\title{
Comparación de la efectividad de la ligadura vs esclerosis endoscópica en pacientes con sangrado de várices esofágicas en el Hospital Hernán Henríquez de Temuco: estudio de cohortes comparativas
}

\author{
EDDY RÍOS ${ }^{1}$, ARMANDO SIERRALTA $^{1}$, MARIGRACIELA ABARZÚA ${ }^{a}$, \\ JOAQUÍN BASTÍAS ${ }^{\mathrm{a}}$, MARÍA INÉS BARRA ${ }^{\mathrm{a}}$
}

\section{Comparison of band ligation with sclerotherapy for the treatment of bleeding esophageal varices}

Background: Endoscopic band ligation is the treatment of choice for bleeding esophageal varices. However it is not clear if this procedure is associated with less early and late mortality than sclerotherapy. Aim: To assess rates of re-bleeding and mortality in cohorts of patients with bleeding esophageal varices treated with endoscopic injection or band ligation. Patients and Methods: Analysis of medical records and endoscopy reports of two cohorts of patients with bleeding esophageal varices, treated between 1990 and 2010. Of these, 54 patients were treated with sclerotherapy and 90 patients with band ligation. A third cohort of 116 patients that did not require endoscopic treatment, was included. The mean analyzed follow up period was 2.5 years (range 1-16). Collection of data was retrospective for patients treated with sclerotherapy and prospective for patients treated with band ligation. Rates of re-bleeding and medium term mortality were assessed. Results: During the month ensuing the first endoscopic treatment, re-bleeding was recorded in 39 and $72 \%$ of patients treated with band ligation and sclerotherapy, respectively $(p<0.01)$. The relative risk of bleeding after band ligation was 0.53 (95\% confidence limits 0.39 0.73). Death rates until the end of follow up were 20 and $48 \%$ among patients with treated with band ligation and sclerotherapy, respectively $(p<0.01)$, with a relative risk of dying for patients subjected to band ligation of 0.41 (95\% confidence limits 0.25-0.68). Conclusions: Band ligation was associated with lower rates of re-bleeding and mortality in these cohorts of patients.

(Rev Med Chile 2012; 140: 713-718).

Key words: Esophageal and gastric varices; Hypertension, portal; Sclerotherapy.

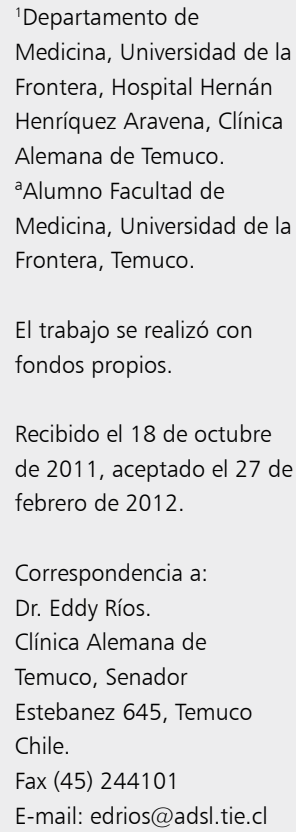

I a hemorragia digestiva alta (HDA) por ruptura de várices esofágicas continúa siendo una de las emergencias más dramáticas de la medicina interna. Hasta $30 \%$ de las personas con cirrosis desarrollan várices ${ }^{1}$, entre $30 \%$ y $70 \%$ de estas sufren de una o más rupturas en el tiempo ${ }^{2}$, ocasionando hemorragias con mortalidad de hasta $30 \%$ para el primer episodio, y en aumento en los siguientes episodios ${ }^{3}$. La tasa de resangrado puede alcanzar de $30 \mathrm{al} 40 \%$ en las primeras seis semanas $^{4}$, y está significativamente relacionada con la mortalidad en este período ${ }^{5}$.

El tratamiento más efectivo fue la esclerosis endoscópica, usando derivados del alcohol ${ }^{6-9}$. En 1988 se describió la técnica de ligadura de várices ${ }^{10}$, la que se expande con el desarrollo de los equipos 
de ligaduras múltiples y se tiene capacidad de detener la hemorragia hasta en $90 \%$ de los casos $^{3,11,12}$.

La literatura en general reporta ventajas del uso de ligadura endoscópica frente a la esclerosis endoscópica en términos de detención de la hemorragia y una menor tasa de resangrado ${ }^{12-14}$, pero las diferencias encontradas no son muy grandes, al punto que la mayoría de las guías recomiendan ambas técnicas indistintamente ${ }^{15-17}$ y existen datos escasos de los efectos a mediano y largo plazo. Una revisión sistemática Cochrane del tema se está realizando en el momento ${ }^{18}$.

El objetivo de este trabajo fue contribuir a aclarar las ventajas o desventajas de un método sobre el otro, especialmente en términos de resangrado, y la mortalidad a mediano plazo de acuerdo a nuestra experiencia.

\section{Material y Métodos}

Doscientos sesenta pacientes fueron diagnosticados con várices esofágicas entre 1990 y 2010, 144 de ellos presentaron hemorragia aguda por las várices esofágicas. Todos fueron seguidos por tiempos variables, a partir del año 1990. El 10\% de ellos se concentraron en la primera década y $90 \%$ entre los años 2000 y 2010. El seguimiento se realizó mediante el estudio de las fichas clínicas y los informes de endoscopia, usando una base de datos especialmente formulada. Las cohortes fueron históricas para el período 1990 al 2007, y concurrentes desde ese año hasta el 2010. El promedio de seguimiento por paciente individual fue de 2,5 años (rango 1-16 años).

Se confeccionaron dos cohortes: La primera con 54 pacientes con várices esofágicas sangrantes tratadas con escleroterapia endoscópica (CE) y la segunda con 90 pacientes similares tratados con ligadura endoscópica (CL). Los criterios de inclusión fueron todos los pacientes que se presentaron en el hospital con hemorragia aguda por várices esofágicas sangrantes, que fueran tratadas ya sea con esclerosis o con ligaduras endoscópicas, y que tuvieran datos completos. Los criterios de exclusión fueron hemorragias por otras causas, prevención primaria o secundaria de várices esofágicas con cualquier método endoscópico, o datos incompletos. Se realizó una tercera cohorte de comparación de 116 pacientes con várices esofágicas que no necesitaron tratamiento endoscópico (CSTE), a fines de comparación. Una pequeña porción de los pacientes tratados con esclerosis fueron tratados también con ligadura en los siguientes episodios de resangrado y sus datos se analizan tanto en conjunto como separadamente.

Se realizó un cálculo post hoc del poder del estudio. Entre las dos cohortes principales, CE y CL, suman 144 pacientes (1,6 ligadura por cada esclerosis), usando el odds ratios de 0,5 obtenido del metaanálisis de Laine ${ }^{20}$ como diferencia teórica, los resultados se pueden leer con un error $\alpha$ de 0,05 , y un error $\beta$ de 0,20 (confiabilidad $95 \%$ y poder $80 \%$ ). El poder aumenta en las comparaciones entre la CL y CSTE.

No se obtuvieron consentimientos informados, ya que las intervenciones fueron las normales para la patología específica y la recogida de datos retrospectiva. No se realizó ningún procedimiento diferente del habitual a causa de este estudio. Las intervenciones fueron realizadas por médicos endoscopistas experimentados de planta. Las esclerosis fueron realizadas con inyecciones intra o paravariaceales de monoetanolamina al 5\%, en ampollas de $5 \mathrm{cc}$. Las ligaduras fueron realizadas con sistemas Cook Medical de 4, 6 ó 10 elásticos. Cada endoscopista decidió en su momento el método y el número de várices intervenidas. Todos los pacientes fueron tratados posteriormente con propranolol, ajustando la dosis según frecuencia cardiaca. El resangrado fue definido como un nuevo episodio de HDA por várices, dentro del mes siguiente. $\mathrm{La}$ mortalidad fue definida como estado vital del paciente al final del seguimiento (año 2010).

La base de datos se construyó mediante códigos que se mantuvieron estables durante todo el estudio. Para el análisis se usó el software Stata 9.0. Las variables cuantitativas se presentan con promedio, desviación estándar y rangos. Las comparaciones usan análisis de $t$ de Student o $U$ test de Mann-Whitney. Las variables discretas se presentan como números absolutos y porcentajes, usando el test de $\chi^{2}$ y el test exacto de Fisher. Las cohortes fueron analizadas con análisis de riesgo relativo con intervalos de confianza, diferencia de riesgos y número necesario a tratar. Los análisis de sobrevida fueron realizados de acuerdo a la técnica de Kaplan Meier. Para el análisis multivariado se realizó un modelo de regresión de Cox utilizando la sobrevida como variable independiente, y las variables ligadura, esclerosis, edad, sexo, uso de propanolol, clasificación de Child $(\mathrm{A}$ y $\mathrm{B}+\mathrm{C})$ y sus interacciones en el modelo. 


\section{Resultados}

Del grupo total de 260 pacientes, 80 fueron mujeres (31\%) y 180 varones $(69 \%)$, relación hombre/mujer de 2,2 a 1. El promedio de edad fue de 56,5 años (DE 13,57, rango 1 a 93 años). La causa de la cirrosis fue el consumo de alcohol en 145 casos (56,9\%), cirrosis post hepatitis viral en $5(2 \%)$, cirrosis autoinmunitaria en $12(4,7 \%)$, y desconocida en $98(38,4 \%)$ probablemente secundaria a esteatosis. De este total, 144 (55,3\%) presentaron sangrado atribuible a las várices como primer diagnóstico mientras que $116(44,7 \%)$ no lo presentaron y las várices fueron diagnosticadas como parte del estudio de su patología. Del total que sangraron, $84(32,3 \%)$ sangraron en una sola oportunidad, mientras que $39(15 \%)$ sangraron 2 veces, $21(9,8 \%)$ tres veces, $11(4,2 \%) 4$ veces y porcentajes menores entre 5 y 12 veces. El grado de severidad en el primer diagnóstico fue de 157 pacientes $(60,4 \%)$ en estadio Child A, $90(34,6 \%)$ en estadio Child B y 13 (5\%) en estadio Child C. La distribución y comparación de todas estas variables para ambas cohortes se muestran en la Tabla 1.

De los 144 pacientes que sangraron por várices en forma aguda, $54(37,5 \%)$ fueron tratadas con esclerosis endoscópica con inyección de monoetanolamina (cohorte de esclerosis CE)y $90(62,5 \%)$, fueron tratadas con ligadura elástica (cohorte de ligadura CL). Un tercer grupo de 116 pacientes no recibieron tratamiento endoscópico (cohorte sin intervención CSTE), ya sea porque las várices fueron diagnosticadas como parte del proceso diagnóstico en $82(70,7 \%)$, o porque el sangrado fue de un origen distinto a las várices en $34(29,3 \%)$.

Treinta y nueve de 54 (72\%) pacientes de la CE tuvieron sangrado post procedimiento dentro del mes posterior a la intervención, comparados con

Tabla 1. Distribución de las variables género, edad, años de seguimiento, etiología y gravedad de las cohortes CE y CL

\begin{tabular}{|c|c|c|c|c|}
\hline Variable & & $\begin{array}{l}\text { Cohorte esclerosis } \\
\text { (CE) } n=54\end{array}$ & $\begin{array}{l}\text { Cohorte ligadura } \\
\text { (CL) } n=90\end{array}$ & $\begin{array}{c}\text { Comparación } \\
\text { CE/CL }\end{array}$ \\
\hline \multirow[t]{2}{*}{ Género } & Hombre & $41(75,9 \%)$ & $64(71,1 \%)$ & $\begin{array}{c}p=0,5 \\
N S\end{array}$ \\
\hline & Mujer & $13(24,9 \%)$ & $26(28,9 \%)$ & $\begin{array}{c}p=0,52 \\
N S\end{array}$ \\
\hline Edad & $\begin{array}{l}\text { Años }(\bar{x} \pm D E) \\
\text { (Rango) }\end{array}$ & $\begin{array}{c}55,2 \pm 14,8 \\
(\text { R 18-96) }\end{array}$ & $\begin{array}{c}54,9 \pm 13,6 \\
(R 21-78)\end{array}$ & $\begin{array}{c}p=0,62 \\
N S\end{array}$ \\
\hline \multirow[t]{4}{*}{ Etiología } & Alcohol & $35(64,8 \%)$ & $53(60,2 \%)$ & $\begin{array}{c}p=0,48 \\
N S\end{array}$ \\
\hline & Post hepatitis & $3(5,6 \%)$ & $2(2,3 \%)$ & $\begin{array}{c}p=0,29 \\
N S\end{array}$ \\
\hline & Auto inmunitaria & $2 \quad(3,7 \%)$ & $7 \quad(7,9 \%)$ & $\begin{array}{c}p=0,32 \\
N S\end{array}$ \\
\hline & Post esteatosis & $14(25,9 \%)$ & $26(29,5 \%)$ & $\begin{array}{c}p=0,70 \\
N S\end{array}$ \\
\hline \multirow[t]{3}{*}{ Estadio } & Child A & $32(59,2 \%)$ & $49(54,4 \%)$ & $\begin{array}{c}p=0,57 \\
N S\end{array}$ \\
\hline & Child B & $21(38,9 \%)$ & $38(42,2 \%)$ & $\begin{array}{c}p=0,79 \\
N S\end{array}$ \\
\hline & Child C & $1 \quad(1,8 \%)$ & $3(3,3 \%)$ & $\begin{array}{c}p=0,60 \\
N S\end{array}$ \\
\hline Seguimiento & $\begin{array}{l}\text { Años }(\bar{x} \pm D E) \\
\text { (Rango) }\end{array}$ & $\begin{array}{l}2,5 \pm 3 \\
(R \quad 1-12)\end{array}$ & $\begin{array}{c}1,57 \pm 0,9 \\
(R 1-5)\end{array}$ & $p=\underset{s}{0,006}$ \\
\hline
\end{tabular}


35 de $90(38,9 \%)$ pacientes de la CL (p $=0,004)$. El riesgo relativo de presentar sangrado luego de ligadura fue de 0,53 (IC 95\% 0,39-0,73) comparado con esclerosis, lo que resulta en un NNT de 3 (se debe someter a 3 pacientes a ligadura en vez de esclerosis para evitar un episodio de re sangrado).

Veintiséis de $54(48 \%)$ pacientes de la CE fallecieron al final del tiempo de seguimiento, $65 \%$ de hemorragia, $31 \%$ de otras complicaciones de la cirrosis y $4 \%$ de causas no relacionadas a la cirrosis, comparados con 18 de 90 pacientes (20\%) en la CL, $40 \%$ de ellos por hemorragia y $60 \%$ de otras complicaciones de la cirrosis. El riesgo relativo de morir luego de ser sometido a ligadura es de 0,41 (IC 95\% 0,25-0,68) comparado con la esclerosis, lo que resulta en un NNT de 3,5 (se debe someter a 3,5 pacientes a ligadura en vez de esclerosis para evitar una muerte). La CSTE tuvo 19 muertos de 116 pacientes $(16,4 \%), 23 \%$ de hemorragia y $76 \%$ de otras causas.

Diecisiete pacientes que fueron tratados con esclerosis, $y$ al volver a sangrar fueron tratados con ligadura, que ya estaba disponible en ese momento. Este grupo puede sesgar los resultados. Para aclarar la magnitud y la dirección del sesgo, el número se restó de ambas cohortes, ajustando a las intervenciones puras de ligadura o esclerosis. Este nuevo análisis muestra que en la CL de 14 de 73 (19\%) pacientes habían muerto al final del seguimiento, mientras que en la CE fallecieron
22 de $37(59,4 \%)$ pacientes ( $\mathrm{p}=0,000)$, con un RR de 0,32 (IC95\% 0,18-0,55, NNT de 2,48). Si sólo se compara esta pequeña cohorte sometida a ambos procedimientos con la CL, el RR es de 1,2 y el NNT de 23, y si se compara con la CE, el RRes de 0,39 , NNT de 2,8, lo que demuestra gráficamente que ambos procedimientos combinados son peores que la ligadura sola, pero mejores que la esclerosis sola.

El análisis de sobrevida de Kaplan Meyer para todas las cohortes (Figura 1) presenta diferencias significativas en la sobrevida de la CL comparada con la CE, mostrando además que el grueso de la mortalidad de ambas cohortes se produce en los primeros 100 meses, para estabilizarse por el resto del seguimiento.

El análisis multivariado de regresión de Cox encontró que de todas las estudiadas, las variables ligadura y esclerosis, más la suma de los pacientes con estadios Child B y C, eran capaces de modificar la sobrevida final (Tabla 2).

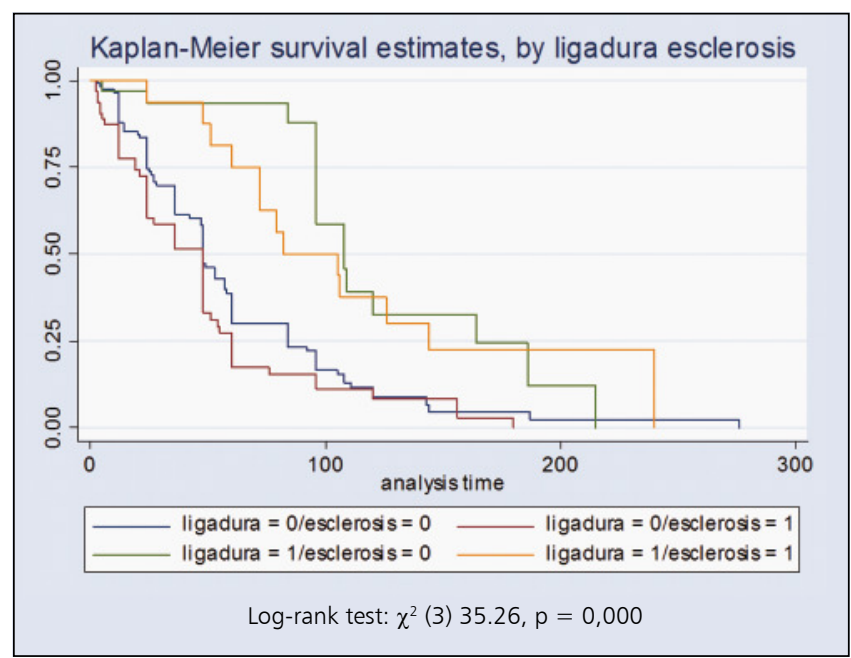

Figura 1. Análisis de sobrevida de las cohortes (ligadura, esclerosis, ambas y ninguna).

Tabla 2. Modelo final de la regresión de Cox con las variables que modifican la mortalidad

\begin{tabular}{|lccccc|}
\hline $\mathbf{t}$ & Coef. & Std. Err. & $\mathbf{z}$ & $\mathbf{P ~ > ~ z ~}$ & [95\% CI] \\
Sobrevida & 37,4391 & & & & \\
Esclerosis &,- 8997443 &, 2137492 & $-4,21$ & 0,000 & $-1,318685-, 4808035$ \\
Ligaduras &, 2320203 &, 1569966 & 1,48 & 0,139 &,$- 0756873-, 539728$ \\
Child B+C &, 3368835 &, 1637964 & 2,06 & 0,040 &, $0158484-, 6579187$ \\
\hline
\end{tabular}

Variables estudiadas: esclerosis, ligaduras, sexo, edad, uso de propranolol, estadíos Child A y suma de Child B+C y sus interacciones. 


\section{Discusión}

El mejor estudio para responder una pregunta de la eficacia de un tratamiento es un ensayo clínico aleatorizado. Un estudio de cohortes como el actual, tiene muchas desventajas por la ausencia de aleatorización y de ciegos, y el carácter retrospectivo de una de ellas. Sin intentar equiparar la calidad de la evidencia obtenida (A2 vs B2), los resultados de la aleatorización se miden en la distribución similar de todos las covariables que no sean los resultados de la intervención. En este estudio, las cohortes estudiadas muestran una distribución similar de todas las variables de interés que pudiera haber desbalanceado los resultados excepto en el tiempo de seguimiento (Tabla 1). Estas variables se comportan tal y como si hubieran sido aleatorizadas exitosamente. La explicación de este fenómeno es que la patología y los pacientes en estudio son similares dentro del período de tiempo estudiado. En cuanto al posible sesgo introducido por la falta de ciegos, al no poder coger uno u otro procedimiento en su momento, ya que la ligadura no estaba aún disponible, este no parece muy importante.

Otro posible sesgo se encuentra en el tiempo del seguimiento. Dada la historia natural y clínica de la enfermedad, es de esperar que mientras más tiempo pase la mortalidad será mayor. Esto puede sesgar los resultados a favor de la ligadura cuya cohorte tuvo como promedio un año menos de seguimiento. La manera final de aclarar este punto es continuar con el seguimiento aproximadamente un año más, y realizar una nueva comparación cuando los tiempos se equiparen. Como dato adicional el análisis de sobrevida muestra que la mortalidad para todos los tipos de tratamiento se produce mayormente en el primer tercio de tiempo post intervención y luego se estabiliza, lo que disminuye este posible sesgo, aunque probablemente no lo elimina (Figura 1).

Si bien las cohortes no son muy grandes, su número alcanza para mantener el error del azar dentro de los límites comúnmente aceptados, y es uno de los que cuenta con mayor $\mathrm{n}$ de todos los estudios publicados ${ }^{13,14}$.

Los resultados obtenidos son válidos con las variables que fueron posibles de estudiar, otras variables como manejo de la HDA en UCI, drogas vasoactivas, profilaxis antibiótica y otros, no se tomaron en cuenta por que en su momento no es- taban disponibles o los datos no fueron suficientes, por lo que su papel en disminuir la mortalidad no puede ser adecuadamente evaluada en este trabajo.

Con las poblaciones estudiadas, se pueden responder varias preguntas. Por un lado se logra obtener un panorama regional claro en relación a la historia clínica de las várices en pacientes portadores de cirrosis. En nuestro medio, con un total de 597 pacientes hospitalizados por cirrosis hepática en la década del 2000 al 2010, 234 de ellos con várices, con seguimiento razonablemente largo y cruce de informaciones, podemos fijar en 39,2\% los porcentajes de presencia de várices $(30 \%)$ en la literatura ${ }^{1}$. El porcentaje de pacientes portadores de várices que sangran en un momento dado en nuestro medio es de 55\%, (30 al 70\%) referido en la literatura ${ }^{1-4}$. El porcentaje de resangrado llega a ser de $67,7,(30-40 \%)$ en la literatura ${ }^{4}$.

Pero el objetivo principal del estudio fue el comparar la eficacia de las dos opciones endoscópicas. La ligadura endoscópica tuvo claras ventajas sobre la esclerosis, en los resultados medidos. La menor tasa resangrado posterior a la intervención (39 vs $72 \%$, RR de 0,53 , NNT 3 ) de la ligadura, y una mortalidad menor ( 20 vs $48 \%$, RR 0,41 , NNT $3,55)$ son importantes y estadísticamente significativas. La proporción de la hemorragia como causa de la muerte también es menor entre ambas cohortes (40 vs $65 \%$ ). Estos resultados son muy similares en su datos numéricos a los obtenidos en el metaanálisis de Laine ${ }^{19}$.

Es interesante el comportamiento de la pequeña cohorte en la que se sobreponen ambos métodos, y que causa un sesgo que cuando es incorporado en el análisis favorece los resultados de la CL, mejorando el RR y el NNT. La tercera cohorte (CSTE), en la que no se hizo ninguna intervención endoscópica en las várices que no sangraron y que, por lo tanto, representaba el espectro menos agresivo de estas, presenta una mortalidad baja, parecida a la ligadura. Esto ha sido previamente descrito en la literatura ${ }^{20,21}$ (Saeed 1997, Umehara 1999).

Dada la historia natural y clínica de la cirrosis, es inevitable que la mortalidad a largo plazo sea la misma para cualquier método de detención de sangrado, por lo que se debe colocar un tiempo límite de corte para juzgar adecuadamente la ventaja de un método sobre otro. A juzgar por la curva de supervivencia, este límite podría ser de alrededor de 80-100 meses. La diferencia del tiem- 
po de seguimiento de ambas cohortes, al ser mayor en la $\mathrm{CE}$, puede estar favoreciendo los resultados encontrados de la ligadura. Excepto el tiempo, los resultados obtenidos parecen depender en gran parte de los procedimientos y no de otras variables, ya que todas las características demográficas, clínicas y de gravedad de la enfermedad se mantuvieron similares y equilibradas entre las cohortes estudiadas. Se debe aclarar que las cohortes fueron de várices tratadas y no de várices erradicadas, lo que no se pudo lograr por muchos motivos.

Este trabajo permite contar con datos propios que ayudan a tomar decisiones de tratamiento, y apoyan las conclusiones de las reuniones de consenso de tratamiento de várices esofágicas, específicamente el punto 3 con datos nacionales, con un nivel de evidencia $2 \mathrm{~b}$, y un grado de recomendación $\mathrm{B}$ favoreciendo la ligadura.

\section{Referencias}

1. Prediction of the first variceal hemorrhage in patients with cirrhosis of the liver and esophageal varices. A prospective multicenter study. North Italian Endoscopic Club for the Study and Treatment of Esophageal Varices. N Engl J Med 1988; 319 (15): 983-9.

2. Burroughs AK. The natural history of varices. J Hepatol 1993; 17 Suppl 2: S10-3. Review.

3. Graham DY, Smith JL. The course of patients after variceal hemorrhage. Gastroenterology 1981; 80 (4): 800-9.

4. Sharara AI, Rockey DC. Gastroesophageal hemorrhage. New England Journal of Medicine 2001; 345: 669-81.

5. D’Amico G, Luca A. Portal hypertension. Natural history. Clinical-hemodynamic correlations. Prediction of the risk of bleeding. Baillieres Clinical Gastroenterology 1997; 11: 243-56.

6. Grace ND. Diagnosis and treatment of gastrointestinal bleeding secondary to portal hypertension. American Journal of Gastroenterology 1997; 92: 1081-91.

7. Goulis J, Burroughs AK. Portal hypertensive bleeding: prevention and treatment. In: McDonald J, Burroughs AK, Feagan B, editor(s). Evidence-based Gastroenterology and Hepatology. London, 1999; 389-426.

8. Sarin SK. Long-term follow-up of gastric variceal sclerotherapy: an eleven year experience. Gastrointest Endosc 1997; 46: 8-14.

9. Trudeau W, Prindiville T. Endoscopic injection sclerosis in bleeding gastric varices.Gastrointest Endosc 1986; 32: 264-8.

10. Steigmann G, Goff GS. Endoscopic esophageal varix ligation: Preliminary clinical experience. Gastrointest Endosc 1988; 34: 113-7.

11. Stiegmann GV, Goff JS, Michaletz-Onody PA, Korula J, Lieberman D, Saeed ZA, et al. Endoscopic sclerotherapy as compared with endoscopic ligation for bleeding esophageal varices. N Engl J Med 1992; 326 (23): 152732.

12. De Franchis R, Primignani M. Endoscopic treatments for portal hypertension. Seminars in Liver Disease 1999; 19: 439-55.

13. Lo GH, Lai KH, Cheng JS, Lin ChK, Huang IS. Emergency banding ligation versus sclerotherapy for the control of active bleeding from esophageal varices. Hepatology 1997; 25: 1101-4.

14. Hashizume M, Ohta M, Ueno K, Tanoue K, Kitano S, Sugimachi K. Endoscopic ligation of esophageal varices compared with injection sclerotherapy: a prospective randomized trial. Gastrointest Endosc 1999; 39: 123-6.

15. Cheung J, Wong W, Zandieh I, Leung Y, Lee SS, Ramji A, et al. Acute management and secondary prophylaxis of esophageal variceal bleeding: a western Canadian survey. Can J Gastroenterol 2006; 20: 531-4.

16. Jalan R, Hayes PC. UK guidelines on the management of variceal haemorrhage in cirrhotic patients. British Society of Gastroenterology. Gut 2000; 46 Suppl 3-4: III1-III15.

17. García-Tsao G, Sanyal AJ, Grace ND, Carey W. Prevention and management of gastroesophageal varices and variceal hemorrhage in cirrhosis. Hepatology 2007; 46: 922-38. doi: 10.1002/hep. 21907

18. D'Amico G, Vitanza ML, Criscuoli VVC, Pagliaro L. Emergency sclerotherapy or band ligation combined with vasoactive drugs for bleeding esophageal varices in cirrhosis (Protocol for a Cochrane Review). In: The Cochrane Library, Issue 02, 2011.

19. Department of Internal Medicine, Royal Infirmary of Edinburgh, Lauriston Place, Edinburgh EH3 9YW, UK.

20. Laine L, Cook D. Endoscopic ligation compared with sclerotherapy for treatment of esophageal variceal bleeding. A meta-analysis. Ann Intern Med 1995; 123 (4): 280-7.

21. Saeed Z, Stiegmann G, Ramírez F, Mathew Reveille R, Goff J. Endoscopic variceal ligation is superior to combined ligation and sclerotherapy for esophageal varices: a multicenter prospective randomized trial. Hepatology 1997; 25: 71-4.

22. Umehara M, Onda M, Tajiri T, Toba M, Yoshida H. Sclerotherapy plus ligation versus ligation for the treatment of esophageal varices: a prospective randomized study. Gastrointest Endosc 1999; 50: 7-12. 